Pierre Damas

Nathalie Layios

Laurence Seidel

Monique Nys

Pierrette Melin

Didier Ledoux

\section{Severity of ICU-acquired pneumonia according to infectious microorganisms}

Received: 21 September 2010

Accepted: 31 March 2011

Published online: 26 May 2011

(C) Copyright jointly held by Springer and ESICM 2011

P. Damas $(\varangle) \cdot$ N. Layios $\cdot$ M. Nys

D. Ledoux

Department of General Intensive Care, University Hospital of Liege,

University of Liege, Domaine Universitaire de Liège, 4000 Liege, Belgium

e-mail: pdamas@chu.ulg.ac.be

Tel.: +32-4-3667495

Fax: +32-4-3668898

\section{Seidel}

Department of Biostatistics,

University Hospital of Liege,

University of Liege, Domaine Universitaire de Liège, 4000 Liege, Belgium

P. Melin

Department of Clinical Microbiology,

University Hospital of Liege,

University of Liege, Domaine Universitaire de Liège, 4000 Liege, Belgium
Abstract Purpose: To assess the severity of intensive care unit (ICU)acquired pneumonia (ICUAP) according to the bacteria involved, classified into seven groups: thirdgeneration cephalosporin-resistant non-fermenting Gram-negative bacilli (resistant C3NF); sensitive C3NF; methicillin-resistant Staphylococcus aureus; methicillin-sensitive Staphylococcus aureus; extended-spectrum beta-lactamase-producing Enterobacteriaceae; Enterobacteriaceae not producing extended-spectrum betalactamase; Haemophilus influenzae and Streptococcus pneumoniae. Methods: Over a 4-year period, sequential organ failure assessment (SOFA) score was prospectively measured daily in 453 adult patients with ICUAP. ICUAP severity was evaluated by the severity of sepsis and by the occurrence of new organ dysfunctions or failures $(\mathrm{OD} / \mathrm{F})$ during ICUAP. Results: Septic shock occurred in $21 \%$ of all cases of ICUAP. The occurrence of new OD/F during ICUAP was similar regardless of the identified microorganism.
These new OD/F represented less than $11 \%$ of SOFAmax, defined as the sum of all OD/F occurring at any time during the ICU stay. There was a significant association between SOFApreICUAP, defined as the sum of all the OD/F occurring before ICUAP, and ICUAP severity. In the multivariate analysis, the type of bacteria was not a risk factor (RF) for occurrence of septic shock and mortality. Age and SOFApreICUAP were RF for the sepsis severity. The ICUAP severity was an RF for ICU mortality. Conclusions: ICUAP was responsible for a minor proportion of OD/F occurring during the ICU stay. Severity of ICUAP was related to clinical status prior to ICUAP, but not to the type of bacteria. ICU mortality depended on the severity of ICUAP.

Keywords ICU · SOFA score · Ventilator-associated pneumonia . Nosocomial infection

\section{Introduction}

ICU-acquired pneumonia (ICUAP) and especially ventilator-associated pneumonia (VAP) are the most common infections in ICU patients and are attributed the highest infection-related mortality [1]. Several factors linked to mortality have been identified: the underlying diseases
$[2,3]$, the severity of the infectious episode (septic shock being an independent risk factor) [3], the type of bacteria (non-fermenting being an independent risk factor) [4, 5], and the appropriateness of antibiotic therapy $[2,6]$. We recently reported global data about organ dysfunction or failure $(\mathrm{OD} / \mathrm{F})$, as assessed by the sequential organ failure assessment (SOFA) score, occurring during ICU-acquired 
infections [7]. The latter contributed only to a minor proportion of OD/F [7]. However, the type of microorganisms involved in the infectious process was not taken into account. The aim of the present study was therefore to extend the analysis of ICUAP by investigating the role played by commonly encountered pathogens, classified according to their difficulty to be treated, i.e., their type and antibiotic resistance.

\section{Materials and methods}

This single-center study was prospectively conducted from 1 January 2004 to 31 December 2007 in a 26-bed ICU at Liege University Hospital, Belgium.

\section{Patients}

All consecutive patients, older than 18 years of age, who stayed for more than $48 \mathrm{~h}$ in the ICU were included in the study. Age, gender, simplified acute physiology score (SAPS) II obtained from the first $24 \mathrm{~h}$ in the ICU [8], and the following underlying diseases were recorded: coronary artery disease, chronic obstructive pulmonary disease, chronic heart failure, cirrhosis, chronic renal failure requiring dialysis, cancer, and hematological malignancies. The severity of the underlying disease was assessed using McCabe and Jackson's classification [9]. The type of admission was classified into traumatic, unscheduled surgery, scheduled surgery, and medical. For patients admitted to the ICU more than once during the same hospital stay, only the first ICU period was recorded.

Infection control policy included continuous surveillance of nosocomial infections, isolation procedures, and routine screening for colonization. Antibiotic treatment was based on local antibiotic guidelines with treatment duration of 8 to 10 days even for non-fermenting organisms [10]. The treatment was considered appropriate when at least one drug from the first empiric antibiotic treatment course was active against the responsible pathogen(s) within $24 \mathrm{~h}$ of clinical diagnosis. During the study period, no selective digestive decontamination was performed and subglottic suction devices were not used. VAP prevention included $30^{\circ}$ head positioning and mouth disinfection with $0.2 \%$ chlorhexidine once per nursing shift. A closed endotracheal suction system, allowing suctioning as frequently as needed, was used for each intubated patient who had to be ventilated for more than $24 \mathrm{~h}$.

The hospital ethics committee approved the study design and the requirement of informed consent was waived because of its epidemiological and noninterventional design.
Definitions

ICUAP was defined as a new and persistent infiltrate on chest radiography occurring more than $48 \mathrm{~h}$ after ICU admission and associated with at least two of the following: fever of above $38.3^{\circ} \mathrm{C}$, leukocyte count of greater than $11,000 / \mathrm{mm}^{3}$, and purulent aspirate. In addition, at least one pathogen had to be identified in significant number by semiquantitative culture of tracheal aspirate. ICUAP was classified according to microorganisms into one of the following seven groups: group 1, third-generation cephalosporin-resistant non-fermenting Gramnegative bacilli; group 2, third-generation cephalosporinsensitive non-fermenting Gram-negative bacilli; group 3, methicillin-resistant Staphylococcus aureus (MRSA); group 4, methicillin-sensitive Staphylococcus aureus (MSSA); group 5, extended-spectrum beta-lactamase (ESBL)-producing Enterobacteriaceae; group 6, Enterobacteriaceae not producing ESBL; group 7, Haemophilus influenzae and Streptococcus pneumoniae. If two or more pathogens were found, the corresponding ICUAP episode was classified according to the most difficult to treat pathogen.

\section{Severity of illness}

The severity of ICUAP was assessed by two methods: first, using the criteria for sepsis and organ failure as defined by the American College of Chest Physicians/ Society of Critical Care Medicine (ACCP/SCCM) conference [11]; secondly, using the SOFA score and several derived indices [7, 12, 13]. According to the ACCP/ SCCM conference recommendation [11] only new or worsened $\mathrm{OD} / \mathrm{F}$ were considered as related to infection and used to determine sepsis severity. For instance, a patient with cardiogenic shock due to myocardial infarction who developed a VAP would have been classified as simple sepsis if the degree of OD/F remained unchanged in terms of specific clinical parameters and need for organ support. In the absence of other obvious explanations, OD/F occurring within $24 \mathrm{~h}$ preceding the clinical diagnosis of an infection were attributed to this infection and taken into account for sepsis severity assessment. Patients suffering from sepsis or simple infection were grouped together as proposed by Alberti et al. and classified as having "simple sepsis" [14].

The SOFA score was calculated daily during the ICU stay following its original description [12]. For the cardiovascular system, a SOFA score of 0 was recorded if vasopressors were used to optimize cerebral perfusion (e.g., in neurotrauma patients). For the central nervous system, the Glasgow coma scale (GCS) was assessed without sedation. In cases of mandatory sedation, the GCS was considered equal to that observed before sedation, usually $15 / 15$. We calculated the SOFAmax, defined 


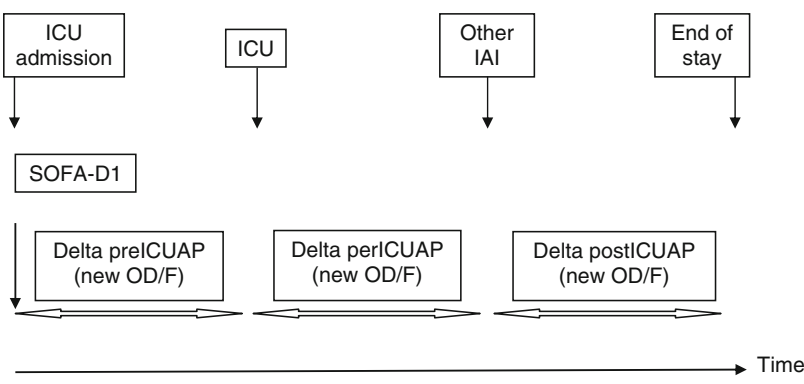

SOFA preICUAP (sum of all OD/F)

SOFAmax (sum of all OD/F during ICU stay) = SOFA-D1 + all deltas

Fig. 1 Calculation of SOFAmax and others derived scores. The ICU stay is divided into three time intervals according to the occurrence of intensive care unit-acquired pneumonia (ICUAP) and possibly other ICU-acquired infection (IAI) after ICUAP. SOFAD1 is the sequential organ failure assessment score of the first day. The deltapreICUAP, deltaperICUAP, and deltapostICUAP are the new organ dysfunctions or failures $(\mathrm{OD} / \mathrm{F})$ occurring during the corresponding time interval. Therefore SOFAmax is the sum of SOFA-D1 and the three delta values. SOFA preICUAP and SOFA perICUAP are the sum of all $\mathrm{OD} / \mathrm{F}$ existing during the corresponding time interval. SOFA sequential organ failure assessment

by Moreno et al. [13] as the sum of the worst scores for each organ component observed at any time during the ICU stay. The SOFAmax was then split into different components derived from daily SOFA calculation (Fig 1): SOFA for the first ICU day, deltapreICUAP calculated as the sum of new OD/F observed before the occurrence of ICUAP, deltaperICUAP corresponding to new OD/F related to the episode of ICUAP, and deltapostICUAP corresponding to new episodes of infection after ICUAP. Severity of ICUAP was, hence, evaluated by the corresponding deltaperICUAP. Furthermore, severity was also assessed by calculating SOFAperICUAP which was the sum of the worst scores observed during the usual 8-10 days' treatment of ICUAP and comparing it to a SOFApreICUAP, which, in the same way, was the sum of the worst scores observed before the occurrence of ICUAP (Fig 1). SOFApreICUAP is therefore the sum of SOFA for the first day plus deltapreICUAP, and SOFAmax is the sum of SOFA for the first day plus deltapreICUAP, deltaperICUAP, and deltapostICUAP.

Bacterial identification, susceptibility testing, and detection of ESBL

Gram-negative bacilli and staphylococcal isolates had been identified and tested for susceptibility by the Vitek 2 system using GN, GP, and AST cards (bio-Merieux,
France). The presence of ESBL in Enterobacteriaceae was screened by the double disk synergy test with three different cephalosporin substrates (cefotaxime, ceftazidime, and cefepime) and clavulanate or by ESBL E tests [15]. Other types of isolates such as Streptococcus pneumoniae were identified by using the API System, conventional tests, and agglutination tests. For these isolates, antimicrobial susceptibility testing was performed by disc diffusion according to CLSI recommendations and minimum inhibitory concentrations (MICs) were obtained by the E test method (ABBiodisc, Sweden). The $\mathrm{E}$ test was performed in accordance with the manufacturer's instructions.

\section{Statistical analysis}

For continuous variables, results are given as mean \pm standard deviation or as median with interquartile range (IQR) when normality was not verified. Categorical variables are presented as number and percentages.

Group characteristics were compared using the likelihood chi-square test or Kruskal-Wallis test as appropriate. We compared SOFA scores between groups using the Kruskal-Wallis test and those within groups using the Wilcoxon matched-pairs signed rank test.

Statistical tests were performed using SAS software (version 9.1.3 Service Pack 4, SAS Institute Inc., Cary, NC, USA). Univariate and multivariate logistic regression models were used to assess associations linking established risk factors with the severity of sepsis and ICU mortality. Because severity of sepsis comprises three modalities (simple sepsis, severe sepsis, and septic shock) the logistic analysis followed an ordinal regression. Results were considered significant when $p<0.05$.

\section{Results}

Over the 4-year period, 2,422 patients older than 18 years of age stayed in the ICU for more than $48 \mathrm{~h}$ and accounted for 29,611 ICU days. The median length of ICU stay was 7 days (IQR 4-15). Six hundred and eighty patients $(28.6 \%)$ acquired 1,088 infections during their ICU stay. Among them, 453 developed 595 ICUAP and 205 other infections. Of these 453 patients only 18 were not ventilated at all, the 435 others were ventilated for a median of 18 days (IQR 9-28). Within the 453 patients who contracted ICUAP, $348(76.8 \%)$ had one single episode of ICUAP, 77 (17\%) had 2 episodes, 21 (4.6\%) had 3 episodes, $5(1.1 \%)$ had 4 episodes, and $2(0.4 \%)$ had 5 episodes. Overall, 86/595 (14.5\%) of ICUAP were responsible for a concomitant bacteremia, and 92/595 (15.5\%) were diagnosed together with another infection. 
Table 1 Distribution and characteristics of ICU acquired pneumonia

\begin{tabular}{|c|c|c|c|c|c|c|c|c|}
\hline ICUAP & C3RNF & C3SNF & MRSA & MSSA & $\begin{array}{l}\text { ESBL } \\
\text { prod Enterobact }\end{array}$ & $\begin{array}{l}\text { Enterobact } \\
\text { not prod ESBL }\end{array}$ & $\begin{array}{l}\text { Hae inf, } \\
\text { S pneu }\end{array}$ & $p$ \\
\hline \multicolumn{9}{|l|}{ First episode } \\
\hline Number & 31 & 92 & 27 & 68 & 36 & 162 & 37 & \\
\hline SOFApreICUAP & $12.1 \pm 5$ & $11.3 \pm 4.4$ & $9.9 \pm 4.2$ & $8.3 \pm 3.2$ & $12.8 \pm 4.8$ & $10.4 \pm 4.1$ & $7.6 \pm 3.7$ & $<0.0001$ \\
\hline SOFAperICUAP & $11.3 \pm 5.8$ & $9.6 \pm 5.3$ & $7.9 \pm 4.8$ & $6.7 \pm 3.4$ & $10.1 \pm 5.6$ & $8.0 \pm 4.6$ & $6.2 \pm 3.5$ & $<0.0001$ \\
\hline DeltaperICUAP & $1(0-3.5)$ & $0(0-3)$ & $0(0-2.5)$ & $0(0-1)$ & $0(0-2)$ & $0(0-2)$ & $0(0-1)$ & 0.3823 \\
\hline Simple sepsis & $12(38.7 \%)$ & $54(58.7 \%)$ & $17(63 \%)$ & $51(75 \%)$ & $18(50 \%)$ & $97(59.9 \%)$ & $28(75.7 \%)$ & \\
\hline Severe sepsis & $6(19.4 \%)$ & $14(15.2 \%)$ & $4(14.8 \%)$ & $10(14.7 \%)$ & $10(27.8 \%)$ & $30(18.5 \%)$ & $3(8.1 \%)$ & 0.0279 \\
\hline Shock & $13(41.9 \%)$ & $24(26.1 \%)$ & $6(22.2 \%)$ & $7(10.3 \%)$ & $8(22.2 \%)$ & $35(21.6 \%)$ & $6(16.2 \%)$ & \\
\hline Appropriateness & $21(67.7 \%)$ & $72(78.3 \%)$ & $22(81.5 \%)$ & $60(88.2 \%)$ & $24(66.7 \%)$ & $141(87 \%)$ & $33(89.2 \%)$ & 0.0094 \\
\hline \multicolumn{9}{|c|}{ Subsequent episodes } \\
\hline Number & 40 & 41 & 5 & 5 & 34 & 16 & 1 & \\
\hline Simple sepsis & $21(52.5 \%)$ & $29(70.7 \%)$ & $5(100 \%)$ & $2(40 \%)$ & $22(64.7 \%)$ & $11(68.8 \%)$ & $1(100 \%)$ & \\
\hline Severe sepsis & $6(15 \%)$ & $7(17.1 \%)$ & 0 & $1(20 \%)$ & $5(14.7 \%)$ & $3(18.8 \%)$ & 0 & 0.4569 \\
\hline Shock & $13(32.5 \%)$ & $5(12.2 \%)$ & 0 & $2(40 \%)$ & $7(20.6 \%)$ & $2(12.5 \%)$ & 0 & \\
\hline
\end{tabular}

ICUAP intensive care unit-acquired pneumonia, C3RNF thirdgeneration cephalosporin-resistant non-fermenting Gram-negative bacilli, C3SNF third-generation cephalosporin-sensitive non-fermenting Gram-negative bacilli, ESBL prod Enterob extendedspectrum beta-lactamase-producing Enterobacteriaceae, Enterobact not prod ESBL Enterobacteriaceae not producing extended-spectrum beta-lactamase, MRSA methicillin-resistant Staphylococcus aureus,
MSSA methicillin-sensitive Staphylococcus aureus, Hae inf, S pneu Haemophilus influenzae and Streptococcus pneumoniae, SOFA sequential organ failure assessment, SOFApreICUAP sum of all the organ dysfunctions/failures occurring before ICUAP, $S O F$ AperICUAP sum of all the organ dysfunctions/failures occurring during ICU stay

shows the partition of SOFAmax according to the time interval during which OD/F occurred, taking only the first episode of ICUAP into account. The SOFA from 1st day in the ICU accounted for $61.4 \pm 22.9 \%$ of the SOFAmax, deltapreICUAP accounted for $25.7 \pm$ $20.8 \%$ of SOFAmax, and deltaperICUAP represented $10.2 \pm 14.6 \%$ of SOFAmax. These proportions were not different between groups.

Table 2 shows that the patients' characteristics classified according to the microorganisms causing their first ICUAP episode were very different in terms of age, past medical history, and lengths of ICU and hospital stay. This was also shown for the severity of acute illness as assessed by the SOFAmax. As expected, the rate of treatment appropriateness was also different. Only the SAPS II score was not statistically significantly different between microorganisms groups.

The multivariate ordinal analysis identified only three statistically significant factors for occurrence of severe sepsis or septic shock: age, presence of a rapidly fatal disease, and SOFApreICUAP. The type of microorganism was not considered as significant (Table 3). Considering ICU mortality (Table 4), the multivariate analysis identified the following as significant risk factors: age, the presence of ultimately or rapidly fatal disease, and the severity of ICUAP. Neither the type of bacteria nor the appropriateness of treatment was retained in the analysis; this was also true for hospital mortality (data not shown). On the other hand, scheduled surgery appeared to be a protective clinical entity. 
Table 2 Characteristics of patients

\begin{tabular}{|c|c|c|c|c|c|c|c|c|}
\hline & $\begin{array}{l}\text { Group } 1 \\
\text { C3RNF }\end{array}$ & $\begin{array}{l}\text { Group } 2 \\
\text { C3SNF }\end{array}$ & $\begin{array}{l}\text { Group } 3 \\
\text { MRSA }\end{array}$ & $\begin{array}{l}\text { Group } 4 \\
\text { StaAur }\end{array}$ & $\begin{array}{l}\text { Group } 5 \\
\text { ESBL }\end{array}$ & $\begin{array}{l}\text { Group } 6 \\
\text { Enterobacteriaceae }\end{array}$ & $\begin{array}{l}\text { Group } 7 \\
\text { Other }\end{array}$ & $p$ \\
\hline $\mathrm{er}$ & 31 & 92 & 2 & 68 & 3 & 162 & 37 & \\
\hline Age & $(41-78)$ & $68(50-78)$ & $66(55-76)$ & $63(48-76)$ & $65(55-76)$ & $63(5$ & $53(45-66)$ & 0.0065 \\
\hline Female sex & $(19.4 \%)$ & $35(38.0 \%)$ & $7(25.9 \%)$ & $25(36.8 \%)$ & $8(22.2 \%)$ & $40(2$ & $5(13.5 \%)$ & 0.0322 \\
\hline Medical & $14(45.2 \%)$ & $38(41.3 \%)$ & $9(33.3 \%)$ & $18(26.5 \%)$ & $15(41.7 \%)$ & $39(24.1 \%)$ & $5(13.5 \%)$ & $=0.0001$ \\
\hline Scheduled surgery & $7(22.6 \%)$ & $18(19.6 \%)$ & $10(37.0 \%)$ & $6(8.8 \%)$ & $6(16.7 \%)$ & $27(16$. & $4(10.8 \%)$ & \\
\hline Emergency sur & $9(29.0 \%)$ & $22(23.9 \%)$ & $4(14.8 \%)$ & $24(35.3 \%)$ & $12(33.3 \%)$ & $55(34.0 \%)$ & $10(27.0 \%)$ & \\
\hline Trauma & $1(3.2 \%)$ & $14(15.2 \%)$ & $4(14.8 \%)$ & $20(29.4 \%)$ & $3(8.3 \%)$ & $41(25.3 \%)$ & $18(48.6 \%)$ & \\
\hline Ultimately fatal & $12(38.7 \%)$ & $22(23.9 \%)$ & $8(29.6 \%)$ & $9(13.2 \%)$ & $15(41.7 \%)$ & $37(22.8 \%)$ & $3(8.1 \%)$ & \\
\hline Rapidly fatal & $8(25.8 \%)$ & $9(9.8 \%)$ & $4(14.8 \%)$ & $1(1.5 \%)$ & $4(11.1 \%)$ & 13 & $0(0 \%)$ & $<0.0001$ \\
\hline hospitalization & $23(74.2 \%)$ & $33(35.9 \%)$ & $9(33.3 \%)$ & $15(22.1 \%)$ & $17(47.2 \%)$ & $3 \%)$ & $4(10.8 \%)$ & $<0.0001$ \\
\hline Ho & 4 & $2(0$ & 0( & & 11) & 1 & 1) & 0.0071 \\
\hline $\begin{array}{l}\text { ICU delay before } \\
\text { ICUAP }\end{array}$ & $10(7-19)$ & $9(6-14)$ & $9(6-12)$ & $5(4-7)$ & $-23)$ & $9(5$ & $6(4-7)$ & $<0.0001$ \\
\hline ICU LOS & 1) & & & & & & $-26)$ & $<0.0001$ \\
\hline Hospital LOS & $42(33-70)$ & $44(31-71)$ & $32(17-52)$ & $32(17-52)$ & $57(39-72)$ & $47(30$ & $32(24-49)$ & \\
\hline Infection on admission & $23(74.2 \%)$ & $39(42.4 \%)$ & $7(25.9 \%)$ & $4(5.9 \%)$ & $26(72.2 \%)$ & $62(38.3 \%)$ & $2(5.4 \%)$ & $<0.0001$ \\
\hline SAPS II & $48.4 \pm 12.3$ & $45.4 \pm 14.9$ & $40.8 \pm 14.2$ & $44.2 \pm 15.3$ & $45.5 \pm 13.4$ & $4308 \pm 12.4$ & $39.5 \pm 13.6$ & 0.0738 \\
\hline ICU mort & $14(45.2 \%)$ & $34(37.0 \%)$ & $7(25.9 \%)$ & $19(27.9 \%)$ & $16(44.4 \%)$ & $40(24.7 \%)$ & $3(8.1 \%)$ & 0.0025 \\
\hline Hospital mortality & $16(51.6 \%)$ & $51(55.4 \%)$ & $11(40.7 \%)$ & $22(32.4 \%)$ & $20(55.6 \%)$ & $55(34.0 \%)$ & $4(10.8 \%)$ & $<0.0001$ \\
\hline
\end{tabular}

LOS length of stay, other abbreviations as per Table 1

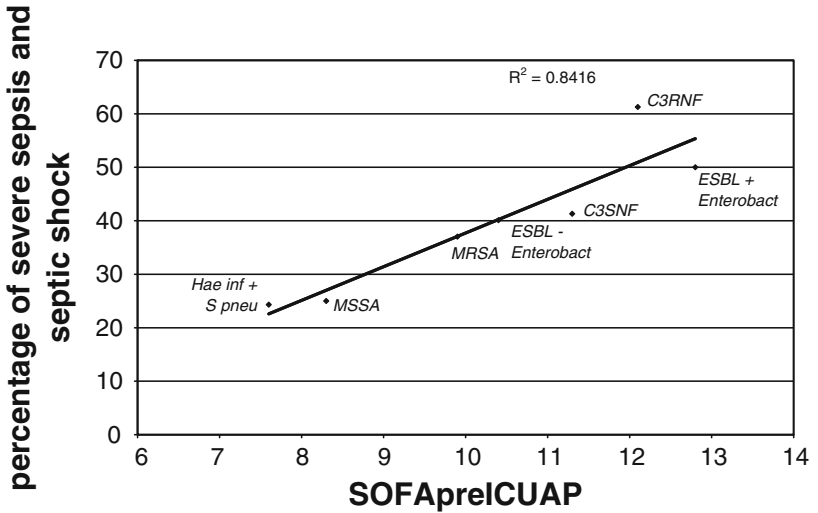

Fig. 2 Relationship between the percentage of severe sepsis and septic shock occurrence during ICU acquired pneumonia classified by their infective microorganisms and the corresponding SOFApreICUAP. ESBL+ Enterobact extended-spectrum betalactamase-producing Enterobacteriaceae, ESBL- Enterobact Enterobacteriaceae not producing extended spectrum beta-lactamase, other abbreviations are defined in Table 1

\section{Discussion}

Determining the contribution of ICUAP to patients' severity of illness is a major challenge. In order to relate new $\mathrm{OD} / \mathrm{F}$ occurring during the ICU stay to clinical events, we measured the SOFA score daily and calculated some derived scores. Whereas SOFAmax measured organ failures for the whole ICU stay, SOFAperICUAP provided information on all the $\mathrm{OD} / \mathrm{F}$ observed during the ICUAP episode only and deltaperICUAP was used to estimate the new OD/F that occurred during ICUAP.

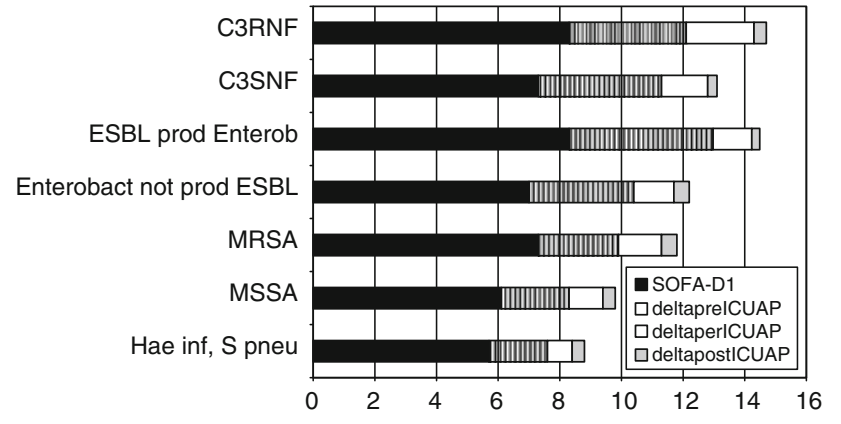

Fig. 3 Partition of the SOFAmax into its constitutive parts. DeltapreICUAP, deltaperICUAP, deltapostICUAP sum of the new organ failures or dysfunctions occuring prior, during or after ICUAP, respectively; other abbreviations are defined in Table 1

These partial SOFA scores were obtained by summing OD/F that occurred during a particular time interval. This is, in our view, a better method to characterize the true severity of illness as compared to the use of mean daily SOFA score. Indeed, the latter approach, by averaging, leads to a loss of relevant information. Interestingly, our approach is similar to SOFAmax calculation which is the best mortality predictor, derived from SOFA score, to date [13].

In order to focus the analysis on the type of microorganism(s), we chose to classify them simply, ranging from the most resistant pathogenic microorganisms to the lesser ones, i.e., non-fermenting Gram-negative bacilli first, Staphylococcus aureus second, Enterobacteriaceae third, and other microbes in fourth place. Then, we separated within the first three groups those presenting 
Table 3 Risk factors for the occurrence of severe sepsis or septic shock: ordinal logistic regression

\begin{tabular}{|c|c|c|c|c|}
\hline \multirow{2}{*}{$\frac{\text { Effect }}{\text { Age }}$} & \multirow{2}{*}{$\begin{array}{l}\text { Point estimate } \\
1.017\end{array}$} & \multicolumn{2}{|c|}{ Confidence limits } & \multirow{2}{*}{$\frac{p \text { value }}{0.0126}$} \\
\hline & & 1.004 & 1.031 & \\
\hline $\operatorname{Sex}(M$ vs. F) & 0.94 & 0.604 & 1.463 & 0.7834 \\
\hline Hospital delay & 0.996 & 0.982 & 1.01 & 0.5376 \\
\hline Previous hospital & 1.031 & 0.665 & 1.599 & 0.8923 \\
\hline Ultimately fat vs. non fatal & 1.544 & 0.964 & 2.473 & 0.0705 \\
\hline Rapidly fatal versus non fatal & 2.042 & 1.001 & 4.165 & 0.0496 \\
\hline SAPS II score & 0.989 & 0.972 & 1.006 & 0.197 \\
\hline \multicolumn{5}{|l|}{ Type of patients } \\
\hline Scheduled surgery versus medical & 1.265 & 0.696 & 2.3 & 0.4411 \\
\hline Emergency surgery versus medical & 0.653 & 0.393 & 1.085 & 0.1002 \\
\hline Trauma versus medical & 0.609 & 0.316 & 1.175 & 0.1391 \\
\hline Infection on admission & 1.247 & 0.775 & 2.007 & 0.3626 \\
\hline SOFApreICUAP & 1.06 & 1.004 & 1.118 & 0.0344 \\
\hline \multicolumn{5}{|l|}{ Type of microorganisms } \\
\hline MSSA versus other & 0.723 & 0.28 & 1.868 & 0.5032 \\
\hline MRSA versus other & 0.845 & 0.278 & 2.57 & 0.7664 \\
\hline Enterob ESBL- versus other & 1.207 & 0.52 & 2.801 & 0.6619 \\
\hline Enterob ESBL+ versus other & 1.263 & 0.426 & 3.745 & 0.6738 \\
\hline C3SNF versus other & 1.117 & 0.451 & 2.767 & 0.8115 \\
\hline C3RNF versus other & 1.05 & 0.63 & 1.748 & 0.8518 \\
\hline Appropriateness & 1.05 & 0.63 & 1.748 & 0.8518 \\
\hline ICUUAPि delay & 0.979 & 0.953 & 1.005 & 0.1138 \\
\hline
\end{tabular}

Enterob ESBL- Enterobacteriaceae not producing extended-spectrum beta-lactamase, Enterob ESBL+ Enterobacteriaceae producing extended-spectrum beta-lactamase, other abbreviations are defined in Table 1

Table 4 Risk factors for ICU mortality: multivariate logistic regression

\begin{tabular}{|c|c|c|c|c|}
\hline \multirow{2}{*}{$\frac{\text { Effect }}{\text { Age }}$} & \multirow{2}{*}{$\begin{array}{l}\text { Point estimate } \\
1.033\end{array}$} & \multicolumn{2}{|c|}{$95 \%$ confidence limits } & \multirow{2}{*}{$\frac{p \text { value }}{0.0004}$} \\
\hline & & 1.015 & 1.052 & \\
\hline Sex (M vs. F) & 0.977 & 0.565 & 1.69 & 0.9344 \\
\hline Hospital delay & 1.009 & 0.993 & 1.025 & 0.2751 \\
\hline Previous hospital & 1.234 & 0.719 & 2.117 & 0.4456 \\
\hline Ultimately fat versus non fatal & 2.359 & 1.335 & 4.168 & 0.0031 \\
\hline Rapidly fatal versus non fatal & 8.796 & 3.538 & 21.865 & $<0.0001$ \\
\hline SAPS II score & 0.996 & 0.975 & 1.017 & 0.7111 \\
\hline \multicolumn{5}{|l|}{ Type of patients } \\
\hline Scheduled surgery versus medical & 0.423 & 0.195 & 0.918 & 0.0296 \\
\hline Emergency surgery versus medical & 0.928 & 0.507 & 1.699 & 0.8088 \\
\hline Trauma versus medical & 0.632 & 0.271 & 1.475 & 0.2891 \\
\hline Infection on admission & 0.771 & 0.422 & 1.408 & 0.3979 \\
\hline SOFApreICUAP & 1.051 & 0.982 & 1.124 & 0.1498 \\
\hline \multicolumn{5}{|l|}{ Severity of ICUAP } \\
\hline Severe sepsis versus simple sepsis & 2.629 & 1.378 & 5.015 & 0.0033 \\
\hline Septic shock versus simple sepsis & 5.392 & 3.011 & 9.655 & $<0.0001$ \\
\hline \multicolumn{5}{|l|}{ Type of microorganisms } \\
\hline MSSA versus other & 3.426 & 0.851 & 13.797 & 0.0831 \\
\hline MRSA versus other & 1.503 & 0.3 & 7.538 & 0.6201 \\
\hline Enterob ESBL- versus other & 1.54 & 0.401 & 5.921 & 0.5294 \\
\hline Enterob ESBL+ versus other & 2.836 & 0.584 & 13.767 & 0.1959 \\
\hline C3SNF versus other & 2.386 & 0.593 & 9.602 & 0.2208 \\
\hline C3RNF versus other & 2.008 & 0.401 & 10.048 & 0.3961 \\
\hline Appropriateness & 1.048 & 0.557 & 1.97 & 0.8854 \\
\hline ICUAP delay & 0.995 & 0.961 & 1.03 & 0.7737 \\
\hline
\end{tabular}

Abbreviations are defined in Tables 1 and 3

antibiotic resistance thus leading to seven different Table 2, we found the expected differences between mutually exclusive ICUAP entities. It should be noted groups in terms of age, past medical history, underlying that this is derived from common clinical practice on diseases, delay between hospitalization and ICU admisinitiation of empiric antibiotic treatment. As shown in sion, delay between ICU admission and occurrence of 
ICUAP, corresponding to what is already known in the literature [16, 17].

The originality of the present study lies in the description of OD/F over the entire ICU course using a score developed for that purpose [12]. Thus, it differs from recent studies on severity of sepsis or VAP that measured SOFA score [18], a new score called VAP-PIRO [19], or the LOD score [20] from the day of infection. In our study, we found that delta SOFA related to ICUAP explained only $10.3 \%$ of the SOFAmax which was by far lower than the contribution of SOFApreICUAP (the sum of SOFA-D1 and deltapreICUAP), which accounted for $87.5 \%$ of SOFAmax (Fig. 3). This corresponds to our previous results [7]. This surely puts the relationship between VAP and its attributable mortality into a new perspective and may also explain why prevention measures of VAP did not have a favorable impact on mortality in many studies, as recently discussed by Klompas [21].

Twenty-one percent of ICUAP were severe enough to cause septic shock. Table 1 suggests that severity may depend on the type of bacteria involved in the infectious process because septic shock seemed to occur more often when third-generation cephalosporin-resistant non-fermenting Gram-negative bacilli were encountered. Interestingly, once again, severity of ICUAP, assessed by the occurrence of septic shock or severe sepsis, seemed to be mainly related to patients' clinical status before ICUAP, as shown by Fig. 2. The determination coefficient $r^{2}$ shows that $84 \%$ of the variability in the incidence of shock or severe sepsis between the different groups of microorganisms is actually explained by the variability of the SOFApreICUAP. We have to insist here on the fact that this is not due to a linkage of measures because OD/F already existing before ICUAP could not be used to define the severity of ICUAP. The multivariate ordinal logistic analysis corroborated this observation by demonstrating that age and SOFApreICUAP were risk factors for the occurrence of severe sepsis and septic shock. One will note that such an approach mainly attributes the severity of sepsis to preexisting OD/F. Therefore, our assumption is not in concordance with the currently accepted theory of sepsis in which an exacerbated host response to infection is solely incriminated [22, 23].
The multivariate analysis did not show infection by any particular group of microorganisms to be a risk factor for ICU mortality. Although many studies found that antibiotic treatment appropriateness is an independent risk factor for mortality [2, 6, 24], the present study did not confirm this finding, like others [25].

Nevertheless, the present study has weaknesses: its single-center design may be criticized. However, our data and patients' characteristics were not different from other large recent epidemiological studies [26, 27]. Another flaw concerns the lack of systematic quantitative cultures as part of the ICUAP definition. However this point is still controversial in the literature [28, 29]. A recent prospective study could not prove the usefulness of quantitative cultures [30]. Also, the ICUAP rates we observed (20/1,000 days or 35/1,000 ventilator days) may appear quite high, but even higher rates are not uncommon in tertiary referral centers [31]. As a matter of fact, the exact same rate was recently corroborated and published in the control group of a randomized controlled trial on VAP and subglottic suction [32]. Magret et al. [33] very recently reported the incidence of VAP in 2,436 patients ventilated for more than $48 \mathrm{~h}$ in 27 ICU from nine European countries: they found 465 patients who developed VAP, thus close to the present figures.

\section{Conclusion}

The severity of ICUAP did not seem to depend on the types of bacteria involved. ICUAP contributed to a minor proportion of $\mathrm{OD} / \mathrm{F}$ occurring during the ICU stay but was responsible for severe sepsis or septic shock in 38\% of the cases. Patients' clinical condition prior to ICUAP was an important factor for infection severity. The high number of OD/F preceding ICUAP and the relationship between $\mathrm{OD} / \mathrm{F}$ and the severity of ICUAP put the relationship between ICUAP and its attributable mortality into a new perspective. The SOFA score provides us with a good tool to assess the impact of events occurring during the ICU stay, especially in the setting of infection.

\section{References}

1. Vincent JL, Bihari DJ, Suter PM, Bruining HA, White J, Nicolas-Chanoin MH, Wolff M, Spencer RC, Hemmer M (1995) The prevalence of nosocomial infection in intensive care units in Europe. Results of the European Prevalence of Infection in Intensive Care (EPIC) Study. EPIC International Advisory Committee. JAMA 274:639-644
2. Jiménez P, Torres A, Rodríguez-Roisin R, de la Bellacasa JP, Aznar R, Gatell JM, Agustí-Vidal A (1990) Incidence, risk and prognosis factors of nosocomial pneumonia in mechanically ventilated patients. Am Rev Resp Dis 142:523-528
3. Fagon JY, Chastre J, Vuagnat A, Trouillet JL, Novara A, Gibert C (1996) Nosocomial pneumonia and mortality among patients in intensive care unit. JAMA 275:866-869

4. Kollef MH, Silver P, Murphy DM, Trovillion E (1995) The effect of lateonset ventilator associated pneumonia in determining patient mortality. Chest 108:1655-1662 
5. Fagon JY, Chastre J, Hance AJ, Montravers P, Novara A, Gibert C (1993) Nosocomial pneumonia in ventilated patients: a cohort study evaluating attributable mortality and hospital stay. Am J Med 94:281-288

6. Kollef MH, Bock KR, Richards RD, Hearns ML (1995) The safety and diagnostic accuracy of minibronchoalveolar lavage in patients with suspected ventilator-associated pneumonia. Ann Intern Med 122:743-748

7. Damas P, Ledoux D, Nys M, Monchi M, Wiesen P, Beauve B, Preiser JC (2008) Intensive care unit acquired infection and organ failure. Intensive Care Med 34:856-864

8. Le Gall JR, Lemeshow S, Saulnier F (1993) A new simplified acute physiology score (SAPS II) based on a European/North American multicenter study. JAMA 270:2957-2963

9. McCabe WR, Jackson GG (1962) Gram-negative bacteremia, aetiology and ecology. Arch Intern Med 110:845-853

10. Chastre J, Wolff M, Fagon JY, Chevret S, Thomas F, Wermert D, Clementi E, Gonzalez J, Jusserand D, Asfar P, Perrin D, Fieux F, Aubas S, Pneum A, Trial Group (2003) Comparison of 8 vs 15 days of antibiotic therapy for ventilator-associated pneumonia in adults: a randomized trial. JAMA 290:2588-2598

11. American College of Chest Physicians/ Society of Critical Care Medicine (1992) American College of Chest Physicians/Society of Critical Care Medicine Consensus Conference: definitions for sepsis and organ failure and guidelines for the use of innovative therapies in sepsis. Crit Care Med 20:864-874

12. Vincent JL, Moreno R, Takala J, Willatts S, De Mendonça A, Bruining H, Reinhart CK, Suter PM, Thijs LG (1996) The SOFA (sepsis-related organ failure assessment) score to describe organ dysfunction/failure. On behalf of the Working Group on Sepsis-Related Problems of the European Society of Intensive Care Medicine. Intensive Care Med 22:707-710

13. Moreno R, Vincent JL, Matos R, Mendonça A, Cantraine F, Thijs L, Takala J, Sprung C, Antonelli M, Bruining H, Willatts S (1999) The use of maximum SOFA score to quantify organ dysfunction/failure in intensive care. Results of a prospective, multicentre study. Working Group on Sepsis Related Problems of the ESICM. Intensive Care Med 25:686-696
14. Alberti C, Brun-Buisson C, Goodman SV, Guidici D, Granton J, Moreno R, Smithies M, Thomas O, Artigas A, Le Gall JR, European Sepsis Group (2003) Influence of systemic inflammatory response syndrome and sepsis outcome of critically ill infected patients. Am J Respir Crit Care Med 168:77-84

15. Brown DF, Andrews J, King A, MacGowan AP (2000) Detection of extended-spectrum beta-lactamases with Etest and double disc potentialisation methods. J Antimicrob Chemother 46:327-328

16. American Thoracic Society; Infectious Diseases Society of America (2005) Guidelines for the management of adults with hospital-acquired, ventilator-associated, and healthcareassociated pneumonia. Am J Respir Crit Care Med 171:388-416

17. Chastre J, Fagon JY (2002) Ventilatorassociated pneumonia. Am J Respir Crit Care Med 165:867-903

18. Vincent JL, Sakr Y, Sprung CL, Ranieri VM, Reinhart K, Gerlach H, Moreno R, Carlet J, Le Gall JR, Payen D, Sepsis Occurrence in Acutely Ill Patients Investigators (2006) Sepsis in European intensive units: results of the SOAP study. Crit Care Med 34:344-353

19. Lisboa T, Diaz E, Sa-Borges M, Socias A, Sole-Violan J, Rodríguez A, Rello J (2008) VAP-PIRO: a tool for predicting ICU mortality and health care resources use in ventilator-associated pneumonia. Chest 134:1208-1216

20. Nguile-Makao M, Zahar JR, Français A, Tabah A, Garrouste-Orgeas M, Allaouchiche B, Goldgran-Toledano D, Azoulay E, Adrie C, Jamali S, Clec'h C, Souweine B, Timsit JF (2010) Attributable mortality of ventilatorassociated pneumonia: respective impact of main characteristics at ICU admission and VAP onset using conditional logistic regression and multi-state models. Intensive Care Med 36:781-789

21. Klompas M (2009) The paradox of ventilator-associated pneumonia prevention measures. Crit Care 13:315

22. Clinel I, Opal SM (2009) Molecular biology of inflammation and sepsis: a primer. Crit Care Med 37:291-304

23. Carlet J, Cohen J, Calandra T, Opal SM, Masur H (2008) Sepsis: time to reconsider the concept. Crit Care Med 36:964-966

24. Melsen WG, Rovers MM, Bonten MJM (2009) Ventilator associated pneumonia and mortality: a systematic review of observational studies. Crit Care Med 37:2709-2710
25. Leroy O, Meybeck A, d'Escrivan T, Devos P, Kipnis E, Georges H (2003) Impact of adequacy of initial antimicrobial therapy on the prognosis of patients with ventilator-associated pneumonia. Intensive Care Med 29:2170-2173

26. Alberti C, Brun-Buisson C, Burchardi H, Martin C, Goodman S, Artigas A, Sicignano A, Palazzo M, Moreno R, Boulmé R, Lepage E, Le Gall R (2002) Epidemiology of sepsis and infection in ICU patients from an international multicentre cohort study. Intensive Care Med 28:108-121

27. Brun-Buisson C, Meshaka P, Pinton P, Vallet B, EPISEPSIS Study Group (2004) EPISEPSIS: a reappraisal of the epidemiology and outcome of severe sepsis in French intensive care units. Intensive Care Med 30:580-588

28. Lisboa T, Rello J (2008) Diagnosis of ventilator-associated pneumonia: Is there a gold standard and a simple approach? Curr Opin Infect Dis 21:174-178

29. Niederman M (2010) The argument against using quantitative cultures in clinical trials and for the management of ventilator-associated pneumonia. Clin Infect Dis 51:593-599

30. The Canadian Critical Care Trials Group (2006) A randomized trial of diagnostic techniques for ventilatorassociated pneumonia. N Engl J Med 355:2619-2630

31. Eggimann P, Hugonnet S, Sax H, Touveneau S, Chevrolet JC, Pittet D (2003) Ventilator-associated pneumonia: caveats for benchmarking. Intensive Care Med 29:2086-2089

32. Lacherade JC, De Jonghe B, Guezennec P, Debbat K, Hayon J, Monsel A, Fangio P, Appere de Vecchi C, Ramaut C, Outin H, Bastuji-Garin S (2010) Intermittent subglottic secretion drainage and ventilator-associated pneumonia : a multicenter trial. Am J Resp Crit Care Med. 182:910-917. doi: 10.1164/rccm.200906-0838OC

33. Magret M, Amaya-Villar R, Garnacho J, Lisboa T, Díaz E, Dewaele J, Deja M, Manno E, Rello J, EU-VAP/CAP Study Group (2010) Ventilator associated pneumonia in trauma patients is associated with lower mortality. Results from EU-VAP study. J Trauma 69:849-854 